\title{
Effects of Modified Simultaneous Unipolar Saline-Irrigated Radiofrequency Ablation in Patients with Atrial Fibrillation Combined with Mitral Valve Disease
}

\author{
Bo Jiang* \\ Ji-Wei Gu* \\ Yan-Yan Song \\ Lei Bai \\ Xu-Dong Liu \\ Yu-jing Zhang \\ Ming-Liang Li \\ Jian Yang \\ Li Liu \\ Yun Wang
}

Department of Cardiovascular Surgery, General Hospital of Ningxia Medical University, Yinchuan, 750004, People's Republic of China

*These authors contributed equally to this work

\begin{abstract}
Objective: To investigate the therapeutic effects of a modified simultaneous unipolar salineirrigated radiofrequency ablation by intracardiac operation under direct vision in patients with mitral valve diseases combined with atrial fibrillation (AF).

Methods: A total of 168 patients with mitral valve diseases combined with AF who underwent unipolar saline-irrigated radiofrequency ablation modified maze procedures were enrolled and divided into the mitral stenosis (MS) group $(\mathrm{n}=87)$ and the mitral insufficiency (MI) group $(\mathrm{n}=81)$.

Results: Those with a left atrium diameter (LAD) $<55 \mathrm{~mm}$ had a better cardioversion effect during the mid-term post-operation than those with a $\mathrm{LAD} \geq 55 \mathrm{~mm}(\mathrm{P}<0.05)$. The cardioversion effect during the mid-term post-operation was better in those with a duration of $\mathrm{AF}<2$ years than those with $\mathrm{AF} \geq 2$ years $(\mathrm{P}<0.05)$. The $\mathrm{LAD}$ reduced significantly during the early postoperative period in the MS group $(\mathrm{P}<0.05)$. Compared with the early postoperative period, LAD further reduced, and the EF increased significantly during the mid-term post-operation $(\mathrm{P}<0.05)$. The LAD reduced significantly during the early postoperative period in the MI group $(\mathrm{P}<0.05)$, together with relatively decreased $\mathrm{EF}(\mathrm{P}<0.05)$. Compared with the early postoperative period, LAD further reduced, and the EF increased significantly during the mid-term post-operation $(\mathrm{P}<0.05)$. The improvement of $\mathrm{LAD}$ in the MI group during the mid-term postoperation was better than that in the MS group $(\mathrm{P}<0.05)$.

Conclusion: The cardioversion effects and the improvement in cardiac function during the mid-term post-operation were good in the radiofrequency ablation by intracardiac operation under direct vision in patients with different mitral valve diseases combined with AF. The cardioversion effects during the early postoperative period and the mid-term post-operation were better in patients with MI than in those with MS.
\end{abstract}

Keywords: mitral stenosis, mitral insufficiency, atrial fibrillation, radiofrequency ablation, therapeutic effect

\section{Introduction}

Atrial fibrillation (AF) is an arrhythmia characterized by rapid and disordered supraventricular electrical activity. ${ }^{1}$ Currently, therapeutic methods for treating AF include surgery, radiofrequency catheter ablation, electrical cardioversion, and drug therapy. However, for patients undergoing surgery for valvular heart disease complicated by $\mathrm{AF}$, concomitant treatment of $\mathrm{AF}$ is particularly important. ${ }^{2}$ The classic maze III procedure for correcting AF, involving the "cutting and suturing" of the atrium, is
Correspondence: Yun Wang Department of Cardiovascular Surgery, General Hospital of Ningxia Medical

University, No. 804 Shengli South Street,

Xingqing District, Yinchuan, 750004,

People's Republic of China

Tel/Fax +86 67430I4

Email wangyun_2Idr@I63.com 
cumbersome. In addition, it increases the duration of the operation and the time on cardiopulmonary bypass, adversely affecting postoperative recovery. ${ }^{3,4}$ The modified Cox III procedure uses energy ablation in place of the cut and suture of the classic maze procedure. It is the most widely used surgical method in the treatment of $\mathrm{AF}^{5}$ and has the advantages of less trauma and a faster recovery time.

The aim of this study was to investigate and evaluate the therapeutic effect of carrying out the unipolar saline-irrigated radiofrequency ablation modified maze procedure concurrently with intracardiac valvular surgery under direct vision for different mitral valve diseases. Patients with mitral stenosis (MS) and mitral insufficiency (MI) combined with AF who underwent this procedure and concomitant valvular surgery between May 2009 and May 2020 in the Cardiovascular Surgery Unit in our hospital were included, and data on the therapeutic effects of this procedure during the early and midterm postoperative periods were collected.

\section{Materials and Methods Clinical Data}

From May 2009 to May 2020, a total of 168 patients with AF and either MS or MI who underwent the unipolar salineirrigated radiofrequency ablation modified maze procedure in the Cardiovascular Surgery Unit of our hospital were enrolled and divided into two groups: there were 87 patients in the MS group, all with rheumatic disease, and 81 patients in the MI group, all with degenerative disease. Methods for the diagnosis of rheumatic or degenerative valvular heart disease included echocardiography and the results of intraoperative and postoperative pathology. AF was confirmed on a 12-lead or 24-hour electrocardiogram (ECG).

The study was conducted in accordance with the Declaration of Helsinki (revised 2013). It was approved by the Ethics Committee of the General Hospital of Ningxia Medical University, and informed consent was taken from all patients.

\section{Surgical Methods}

In all operations, tracheal intubation was carried out following the induction of anesthesia using a combination of intravenous and inhalation agents. Median sternotomy was then performed and the aorta, the superior vena cava and the inferior vena cava were used to establish cardiopulmonary bypass before carrying out the intracardiac operation under direct vision. The right atrium incision was employed, and the modified maze procedure was carried out first. The saline-irrigated unipolar radiofrequency ablation apparatus (the Medtronic Cardioblate ${ }^{\mathrm{TM}} 60880$ type) was used: this included the generator, foot switch, power cord, dispersion electrode cable, and the Cardioblate ${ }^{\mathrm{TM}}$ disposable surgical ablation pen. During the operation, the energy of the ablation device was controlled at 25-30 W/s. The saline irrigation pressure was maintained at approximately $100 \mathrm{mmHg}$, and the irrigation speed was maintained at $180-240 \mathrm{~mL} / \mathrm{h}$. The unipolar radiofrequency ablation pen was used to connect the bilateral pulmonary vein circular ablation line and the two upper poles, between the left lower pulmonary vein and the back of the mitral valve, and between the left upper pulmonary vein orifice and the left atrial appendage. The connecting lines were made between the left ring of the coronary sinus orifice and the mitral valve annulus, between the lower pole of the coronary sinus orifice and the tricuspid valve annulus, and between the coronary sinus orifice and the inferior vena cava. On completion of the modified maze procedure, the left atrial appendage was ligated, and this was followed by surgery of other cardiac valves. ${ }^{15}$

Following the operation, $900 \mathrm{mg}$ of amiodarone was given as an infusion over 24 hours. Amiodarone was then continued orally at a dose of $200 \mathrm{mg}$ three times a day for one week and then reduced to twice daily for a further week, followed by once daily maintenance until three months after the operation. The dosing regimen was then adjusted according to the postoperative cardioversion effect: if the patient remained in sinus rhythm at three months post-surgery, the drug could be discontinued; if the heart rhythm continued to be AF, amiodarone was continued for six months following surgery.

\section{Study Methods}

A retrospective study method was used to evaluate the therapeutic effects of clinical diseases. Depending on the etiology and disease type, the patients were divided into a rheumatic MS group and a degenerative MI group. An ECG was used to detect cardiac rhythm. The ECG results at pre-operation, during the early postoperative period (five to seven days), and the midterm postoperative period (three to six months) were analyzed to evaluate the rate of cardioversion during these periods between the two groups. Echocardiography was used to detect the left atrial diameter (LAD) and the left ventricular ejection fraction (LVEF) at pre-operation and also during the early and midterm postoperative periods, and these results were analyzed. The improvement in LAD and LVEF during the early and midterm postoperative periods and the difference between the two groups were evaluated using intragroup and intergroup comparisons. 


\section{Statistical Analysis}

The relevant data from cardiac color Doppler ultrasonography and ECGs of those that met the inclusion criteria preoperatively and during the early and midterm postoperative periods were listed. The SPSS 20.0 software was used for data analysis. The Chi-square test or Fisher's exact probability method was used for the comparison of enumeration data between the two groups. The measurement data were expressed as the mean \pm standard deviation. The independent sample $t$-test was used for comparing data between the MS and MI groups for those conforming to a normal distribution, and the paired $t$-test was used for the comparison of the preoperative and postoperative data within each group. The non-parametric test (multiple independent sample rank-sum test) was used for data not conforming to a normal distribution. A $P$ value of $<0.05$ was considered to be statistically significant.

\section{Results}

There was no statistically significant difference between the two groups of patients in terms of age, duration of AF, classification of heart function, $\mathrm{LAD}$, or surgical procedures $(P>0.05)$, as shown in Table 1. The comparison of the sinus rhythm cardioversion rates between the two groups during the early and midterm postoperative periods are shown in Tables 2 and 3. Cardioversion rates during the early and midterm postoperative periods in the MI group were better than those in the MS group $(P<0.05)$. In the two groups of patients, those with an $\mathrm{LAD}<55 \mathrm{~mm}$ had a better cardioversion result during the midterm postoperative period than those with an LAD $\geq 55 \mathrm{~mm}(P<$ 0.05), as shown in Table 4. Cardioversion rates during the midterm postoperative period were better in those with $\mathrm{AF}$ of less than two years duration as compared with those in AF for more than two years $(P<0.05)$, as shown in Table 5 . The LAD significantly reduced during the midterm postoperative period, and the ejection fraction (EF) increased in the both groups $(P<0.05)$. The improvement in LAD during the midterm postoperative period in the MI group was better than that in the MS group $(P<0.05)$, while there was no statistically significant difference in $\mathrm{EF}$ between the two groups $(P>0.05)$, as shown in Table 6 .

\section{Discussion}

In patients with valvular heart disease and poor heart function, the heart has endured a large volume and pressure load for a considerable time, and the compensatory ability of the heart has reached its limit. When the patient is active, output requirement increases and the heart

Table I The Comparison of the General Data Between the Two Groups

\begin{tabular}{|c|c|c|c|}
\hline Clinical Characteristics & Mitral Stenosis (MS) Group & Mitral Insufficiency (MI) Group & \\
\hline Gender (male/female) & $28 / 59$ & $49 / 32$ & $P<0.0$ I \\
\hline Age (year) & $54.0 \pm 7.8$ & $56.2 \pm 8.1$ & $P=0.972>0.05$ \\
\hline Duration of atrial fibrillation (year) & $4.8 \pm 4.9$ & 4. $1 \pm 5.7$ & $P=0.909>0.05$ \\
\hline Cardiac function NYHA classification & & & $P>0.05$ \\
\hline II & 25 & 17 & \\
\hline III & 54 & 57 & \\
\hline IV & 8 & 7 & \\
\hline Left atrium diameter $(\mathrm{mm})$ & $57.8 \pm 9.4$ & $56.1 \pm 8.4$ & $P=0.307>0.05$ \\
\hline $\mathrm{EF}(\%)$ & $62.1 \pm 7.4$ & $63.3 \pm 6.9$ & $P=0.392>0.05$ \\
\hline Surgical methods & & & $P>0.05$ \\
\hline MVR+ Modified Maze procedure & 36 & 38 & \\
\hline MVR+TVP+ Modified Maze procedure & 51 & 43 & \\
\hline $\begin{array}{l}\text { Duration of cardiopulmonary } \\
\text { bypass ( } \mathrm{min})\end{array}$ & $127.2 \pm 48.2$ & $136.2 \pm 32.3$ & $P=0.374>0.05$ \\
\hline Duration of aortic block (min) & $76.57 \pm 32.0$ & $81.77 \pm 39.6$ & $P=0.278>0.05$ \\
\hline
\end{tabular}

Notes: Chi-square test or Fisher's exact probability method were used to compare the two groups of count data. Measurement data were expressed as mean \pm standard deviation. 
Table 2 The Comparison of Early Postoperative Sinus Cardioversion Rates Between the Two Groups

\begin{tabular}{|l|l|l|l|l|}
\hline Etiology and Disease Type & Sinus Rhythm & Non-Sinus Rhythm & Total & $\boldsymbol{P}$-value \\
\hline Mitral stenosis (MS) & $46(52.9 \%)$ & $41(47.1 \%)$ & 87 & $P=0.04<0.05$ \\
\hline Mitral insufficiency (MI) & $56(69.1 \%)$ & $25(30.9 \%)$ & 81 & 168 \\
\hline Total & 102 & 66 & \\
\hline
\end{tabular}

Notes: Chi-square test or Fisher's exact probability method was used to compare the rates of early/middle postoperative sinus cardioversion between groups. The effect of cardioversion in the early and mid-postoperative mitral incomplete group was better than that in the mitral stenosis group $(P<0.05)$.

Table 3 The Comparison of Mid-Term Post-Operation Sinus Cardioversion Rates Between the Two Groups

\begin{tabular}{|l|l|l|l|l|}
\hline Etiology and Disease Type & Sinus Rhythm & Non-Sinus Rhythm & Total & $p$-value \\
\hline Mitral stenosis (MS) & $45(60.8 \%)$ & $29(39.2 \%)$ & 74 & $P=0.02<0.05$ \\
\hline Mitral insufficiency (MI) & $49(79.0 \%)$ & $13(21.0 \%)$ & 62 & 136 \\
\hline Total & 94 & 42 & \\
\hline
\end{tabular}

Notes: Chi-square test or Fisher's exact probability method was used to compare the rates of early/middle postoperative sinus cardioversion between groups. The effect of cardioversion in the early and mid-postoperative mitral incomplete group was better than that in the mitral stenosis group $(P<0.05)$.

function becomes decompensated, promoting the occurrence and maintenance of AF. Therefore, it is necessary to correct $\mathrm{AF}$ during mitral valve surgery.

In the present study, the cardioversion rates during the midterm postoperative period increased in both groups, and this was considered to be correlated with the following factors: (1) during the early postoperative period, the inflammation of the myocardium at the surgical site and the disturbance of the myocardial electrical activity may cause shortening of the myocardial refractory period and rhythm instability. The shortening of the refractory period may produce a small diameter feedback loop, which in turn may lead to AF recurrence. Researchers have found that $\mathrm{AF}$ recurrence during this period does not correlate with long-term maintenance of sinus rhythm. ${ }^{6}$ At three months following surgery, the ablation line can scar and form an absolute barrier, which can interrupt the feedback pathway, reduce the AF recurrence rate, and increase the conversion rate to sinus rhythm. ${ }^{7}$
Studies have found that postoperative amiodarone for at least three months can impact on the rhythm instability caused by the postoperative inflammation and thereby reduce the risk of $\mathrm{AF}$ recurrence; ${ }^{8}$ (2) it could be inferred from Table 6 that in the early postoperative period, the LAD was significantly reduced in both groups of patients compared with pre-operation data. The $\mathrm{LAD}$ further reduced during the midterm postoperative period. The difference in LAD recovery during the early postoperative period and the midterm post-operation period might explain the higher conversion rate to sinus rhythm in the midterm postoperative period differs compared with the early postoperative period; and (3) there was no significant improvement in the EF during the early postoperative period when compared with the preoperation status between the two groups. Follow-up at in the midterm postoperative period showed that the EF had increased in both groups relative to the preoperative and early postoperative periods, as shown in Table 6. The

Table 4 The Intra-Group Comparison of Cardioversion Rates in Two Groups of Patients with Different Internal Diameters of the Left Atrium During the Mid-Term Post-Operation

\begin{tabular}{|l|l|l|l|l|l|}
\hline Etiology and Disease Type & Cases & Groups & Sinus Rhythm & Non-Sinus Rhythm & $\boldsymbol{P}$-value \\
\hline Mitral stenosis (MS) & 74 & $\begin{array}{l}\text { Left atrium diameter } \geq 55 \mathrm{~mm} \\
\text { Left atrium diameter }<55 \mathrm{~mm}\end{array}$ & $\begin{array}{l}15(45.5 \%) \\
30(73.2 \%)\end{array}$ & $\begin{array}{l}18(54.5 \%) \\
1 \mathrm{I}(26.8 \%)\end{array}$ & $P=0.018<0.05$ \\
\hline Mitral insufficiency (MI) & 62 & $\begin{array}{l}\text { Left atrium diameter } \geq 55 \mathrm{~mm} \\
\text { Left atrium diameter }<55 \mathrm{~mm}\end{array}$ & $\begin{array}{l}23(67.6 \%) \\
26(92.9 \%)\end{array}$ & $\begin{array}{l}\text { II (32.4\%) } \\
2(7.1 \%)\end{array}$ & $P=0.003<0.05$ \\
\hline
\end{tabular}

Notes: Chi-square test or Fisher's exact probability method was used to compare the cardioversion of patients with different left atrial diameters in the middle stage after surgery between the two groups. The results showed that the left atrial diameters were less than $55 \mathrm{~mm}$, and the sinus rhythm had a good cardioversion rate $(P<0.05)$, which was statistically significant. The interim postoperative cardioversion effect of patients with $L A D<55 \mathrm{~mm}$ was better than that of patients with $L A D \geq 55 \mathrm{~mm}(P<0.05)$. 
Table 5 The Intra-Group Comparison of Cardioversion Rates in Two Groups of Patients with Different Durations of Atrial Fibrillation During the Mid-Term Post-Operation

\begin{tabular}{|l|l|l|l|l|l|}
\hline Etiology and Disease Type & Cases & Groups & Sinus Rhythm & Non-Sinus Rhythm & $\boldsymbol{p}$-value \\
\hline Mitral stenosis (MS) & 74 & $\begin{array}{l}\text { Atrial fibrillation } \geq 2 \text { years } \\
\text { Atrial fibrillation<2years }\end{array}$ & $\begin{array}{l}21(48.8 \%) \\
24(77.4 \%)\end{array}$ & $\begin{array}{l}22(51.2 \%) \\
7(22.6 \%)\end{array}$ & $P=0.016<0.05$ \\
\hline Mitral insufficiency (MI) & 62 & $\begin{array}{l}\text { Atrial fibrillation } 2 \text { 2years } \\
\text { Atrial fibrillation<2years }\end{array}$ & $\begin{array}{l}20(66.7 \%) \\
29(90.6 \%)\end{array}$ & $\begin{array}{l}10(33.3 \%) \\
3(9.4 \%)\end{array}$ & $P=0.029<0.05$ \\
\hline
\end{tabular}

Notes: Chi-square test or Fisher's exact probability method was used to compare the cardioversion of atrial fibrillation in the middle stage after surgery between the two groups. The duration of the disease was less than 2 years, and the sinus rhythm had a good cardioversion rate $(P<0.05)$, which was statistically significant. Patients with a course of atrial fibrillation less than 2 years had better mid-term cardioversion effect than patients with a course of atrial fibrillation more than 2 years $(P<0.05)$.

Table 6 The Comparison of Indicators in the Cardiac Ultrasonography at the Preoperative, Early Postoperative Period and Mid-Term Post-Operation Between Two Groups of Patients

\begin{tabular}{|c|c|c|c|c|}
\hline $\begin{array}{l}\text { Cardiac Ultrasound } \\
\text { Indicators }\end{array}$ & & $\begin{array}{c}\text { Mitral Stenosis (MS) } \\
\text { Group }\end{array}$ & $\begin{array}{c}\text { Mitral Insufficiency (MI) } \\
\text { Group }\end{array}$ & $p$-value \\
\hline Left atrium diameter $(\mathrm{mm})$ & $\begin{array}{l}\text { Pre-operation } \\
\text { Early postoperative } \\
\text { period } \\
\text { p-value } \\
\text { Early postoperative } \\
\text { period } \\
\text { Mid-term post-operation } \\
\text { p-value }\end{array}$ & $\begin{array}{c}57.8 \pm 9.4 \\
49.9 \pm 10.3 \\
P<0.01 \\
49.9 \pm 10.3 \\
46.6 \pm 6.8 \\
P=0.018<0.05\end{array}$ & $\begin{array}{c}56.1 \pm 8.4 \\
47.3 \pm 8.4 \\
P<0.01 \\
47.3 \pm 8.4 \\
42.8 \pm 5.9 \\
P<0.01\end{array}$ & $\begin{array}{l}P=0.307>0.05 \\
P=0.102>0.05 \\
P=0.102>0.05 \\
P=0.012<0.05\end{array}$ \\
\hline EF value (\%) & $\begin{array}{l}\text { Pre-operation } \\
\text { Early postoperative } \\
\text { period } \\
\text { p-value } \\
\text { Early postoperative } \\
\text { period } \\
\text { Mid-term post-operation } \\
\text { p-value }\end{array}$ & $\begin{array}{c}62.1 \pm 7.4 \\
62.5 \pm 6.8 \\
P=0.483>0.05 \\
62.5 \pm 6.8 \\
67.3 \pm 8.6 \\
P<0.01\end{array}$ & $\begin{array}{c}63.3 \pm 6.9 \\
61.2 \pm 9.5 \\
P=0.034<0.05 \\
61.2 \pm 9.5 \\
66.8 \pm 7.2 \\
P<0.01\end{array}$ & $\begin{array}{l}P=0.392>0.05 \\
P=0.425>0.05 \\
P=0.425>0.05 \\
P=0.487>0.05\end{array}$ \\
\hline
\end{tabular}

Notes: Paired $t$ test was used for comparison between horizontal groups and within longitudinal groups at different time points, non-parametric test (rank sum test of multiple independent samples) was used for comparison between groups with normal distribution, and independent sample $t$ test was used for comparison between groups with normal distribution. Compared with the early stage, the middle postoperative LAD in the two groups was significantly reduced, and the EF value was increased $(P<$ 0.05). The improvement of the LAD in the mitral infraction group was better than that in the mitral stenosis group $(P<0.05)$, and there was no statistical difference in the EF value between the two groups $(P>0.05)$.

presence of long-term preoperative valvular disease and perioperative myocardial damage may have caused this and may also lead to disorder of the early postoperative myocardial energy metabolism and ion channel dysfunction. ${ }^{9}$ As the EF did not significantly improve in the short term, this may have had an effect on the cardioversion rates. During the midterm postoperative period, with the relative increase in $\mathrm{EF}$, the cardioversion rates increased.

Results from this study suggest that the cardioversion rates during the early and midterm postoperative periods in patients with MI were better than in those with MS. This may be correlated with the following two factors: (1) the LAD in patients with MI and MS reduced significantly in the early and midterm postoperative periods, and the improvement in LAD during the midterm postoperative period was significantly better in patients with MI than in those with MS (as shown in Table 6), and LAD is a risk factor for postoperative $\mathrm{AF}$ recurrence. The domestic researchers Jankelson et $\mathrm{al}^{10}$ showed that the reversal of left atrial remodeling following mitral valve replacement was significantly better in patients with MI than in patients with MS; (2) in this study, patients in the MS group all had rheumatic diseases while patients in the MI group all had degenerative diseases, and the mechanism of $\mathrm{AF}$ is not the same in both cases. In rheumatic fever, the repeated inflammatory stimulation to the myocardium may aggravate endothelial cell injury. This is more likely to cause 
myocardial structural remodeling and trigger AF with a rapid ventricular rate, thereby worsening hemodynamic abnormalities, reducing cardiac function and impacting on myocardial remodeling, all of which could negatively affect cardioversion rates.

Yao et $\mathrm{al}^{11}$ reported that an increase in the preoperative LAD and a long duration of AF were high-risk factors for AF recurrence following radiofrequency ablation. Prabhu et $\mathrm{al}^{12}$ believed that the inner diameter of the left atrium might directly affect the ablation result, and patients with $\mathrm{AF}$ and a left atrium inner diameter $>55 \mathrm{~mm}$ were likely to experience AF recurrence in the long term. Domestically, Kirchhof et $\mathrm{al}^{13}$ reported that the sinus rhythm cardioversion rate following radiofrequency ablation was significantly higher in patients with AF of less than two years duration as compared with patients in AF for more than two years. In this study, AF duration of less than two years and an LAD of $55 \mathrm{~mm}$ were adopted as cut-off points, and patients with MS and MI were divided into groups. As at least three months is required for the postoperative ablation line to be completely scarred and to form insulation, the AF conversion rate seen within three months of surgery does not entirely reflect the success rate of the surgical treatment of AF. Therefore, the patients were divided into two groups based on AF duration and the LAD. In these groups, those with an LAD $<55 \mathrm{~mm}$ had a better cardioversion effect during the midterm postoperative period than those with an $\mathrm{LAD} \geq 55 \mathrm{~mm}(P<0.05)$. Cardioversion rates during the midterm postoperative period were better in those with AF for less than two years as compared with those with AF of more than two years duration $(P<0.05)$. These findings were consistent with those reported in the literature cited above. Therefore, choosing patients with a short duration of AF and a small LAD prior to surgery improves the success rate of AF treatment during the midterm postoperative period.

This study found that compared with the early postoperative period, during the midterm postoperative period, the LAD in both groups further reduced, and the EF increased significantly. The following factors may collectively explain these findings: (1) the relatively long preoperative duration of the disease may result in severe myocardial injury; (2) the effect of intraoperative injury to the myocardium; and (3) in MI, a large quantity of blood flows back to the left atrium from the left ventricle before systole. Therefore, the EF does not accurately estimate the actual amount of blood pumped into the aorta. Following surgery, when the MI has been corrected, left ventricular preload is reduced and afterload increases. So, although the EF was lower than before surgery, this may not accurately represent the actual blood volume pumped into the aorta. Following replacement of the mitral valve in MS, left ventricular function will not improve substantially in a short time, and the EF does not therefore change significantly. Studies have also shown that the decrease in the inner diameter of the left atrium during the early period of mitral valve surgery is correlated with a decrease in EF postoperatively. ${ }^{14}$ As the observation time extended to the midterm postoperative period, the EF gradually increased.

This study had some limitations. In most patients, conventional ECG rather than 24-hour dynamic ECG monitoring was used to detect the cardiac rhythm. Consequently, some cases of paroxysmal AF may have been missed. Study sample size was small, and the observation period was short. In patients with long-term poor cardiac function before surgery and severe myocardial injury, a certain period is required in order to recover the postoperative cardiac function and sinus rhythm. Therefore, in order to fully and accurately evaluate surgery efficacy, the follow-up period should be extended. Also, the sample sizes in each group were small due to the inability to collect follow-up measurements, and this should be addressed in future studies.

\section{Conclusion}

In summary, this study suggests that following simultaneous radiofrequency ablation for AF by intracardiac operation under direct vision in patients with different mitral valve diseases, cardioversion rates and cardiac function improvement during the midterm postoperative period were good. Cardioversion conversion rates during the early and midterm postoperative periods were better in patients with MI when compared with those with MS. Preoperative selection of patients with a short duration of AF and a small LAD improves the cardioversion rates seen during the midterm postoperative period. To further investigate the long-term effects of the modified maze procedure in the treatment of $\mathrm{AF}$, future studies should be multicenter, involving a large sample size and a long follow-up duration, as well as more scientific research methods.

\section{Acknowledgments}

We are particularly grateful to all the people who have given us help on our article.

\section{Funding}

This study was funded by the Ningxia Natural Science Foundation Project (No. 2020AAC03367). The funding 
body had no role in the design of the study and collection, analysis, and interpretation of data and in writing the manuscript.

\section{Disclosure}

The authors declare that they have no competing interests.

\section{References}

1. Atrial Fibrillation Prevention and Control Expert Working Committee of the Chinese Physicians Association Cardiac Rhythm Committee, Cardiac Electrophysiology and Pacing Branch of the Chinese Medical Association. Atrial fibrillation: current understanding and treatment recommendations-2015. Chin J Cardiac Arrhythmias. 2015;19(5):321-384.

2. Chen S, Schmidt B, Bordignon S, et al. Catheter ablation of atrial fibrillation using ablation index-guided high-power technique: frankfurt AI high-power 15-month follow-up. $J$ Cardiovasc Electrophysiol. 2021;32:616-624. doi:10.1111/jce.14912

3. Lawrance CP, Henn MC, Damiano RJ Jr. Surgery for atrial fibrillation. Heart Fail Clin. 2016;12(2):235-243. doi:10.1016/j. hfc.2015.08.019

4. Philpott JM, Zemlin CW, Cox JL, et al. The ABLATE trial: safety and efficacy of Cox Maze-IV using a bipolar radiofrequency ablation system. Ann Thorac Surg. 2015;100(5):1541-1548. doi:10.1016/j. athoracsur.2015.07.006

5. Ramlawi B, Abu Saleh WK. Surgical ablation of atrial fibrillation. Methodist Debakey Cardiovasc J. 2015;11(2):104-108. doi:10.14797/ mdcj-11-2-104

6. Rivarola EWR, Moura E, Chou M, Feitosa L, Hardy C, Scanavacca M. A novel treatment for esophageal lesions following atrial fibrillation ablation. $J$ Cardiovasc Electrophysiol. 2021;32:713-716. doi:10.1111/jce. 14895
7. Boho A, Stancak B, Misikova S. Efficacy of cryoablation in idiopathic and non-idiopathic atrial fibrillation patients. Bratisl Lek Listy. 2021;122(2):89-94.

8. Grosu AI, Radulescu D, Grosu LC, Pop D. Remodelling in atrial fibrillation: the impact of amiodarone. Cardiovasc J Afr. 2019;30 (3):174-180. doi:10.5830/CVJA-2019-012

9. Ozcan C, Li Z, Kim G, Jeevanandam V, Uriel N. Molecular mechanism of the association between atrial fibrillation and heart failure includes energy metabolic dysregulation due to mitochondrial dysfunction. J Card Fail. 2019;25(11):911-920.

10. Jankelson L, Dai M, Aizer A, et al. Lesion sequence and catheter spatial stability affect lesion quality markers in atrial fibrillation ablation. JACC Clin Electrophysiol. 2021;7:367-377. doi:10.1016/j. jacep.2020.09.027

11. Yao S, Koike H, Fujino T, et al. Role of intracardiac defibrillation during the ablation procedure as a predictor of atrial fibrillation recurrence after catheter ablation. Int Heart J. 2021;62(1):87-94. doi:10.1536/ihj.20-636

12. Prabhu S, Ahluwalia N, Tyebally SM, et al. Long-term outcomes of index cryo-balloon ablation or point by point radiofrequency ablation in patients with atrial fibrillation and systolic heart failure. J Cardiovasc Electrophysiol. 2021. doi:10.1111/jce.14923

13. Kirchhof P, Wegscheider K, Breithardt G. Early rhythm control in atrial fibrillation. Reply N Engl J Med. 2021;384(5):484-485.

14. Thourani VH, George I, Rucinskas K, et al. First in human experience with an epicardial beating heart device for secondary mitral regurgitation. J Thorac Cardiovasc Surg. 2020.

15. Meng X, Wang JG. Radiofrequency ablation under open heart for treatment of atrial fibrillation. Chinese Journal of Thoracic and Cardiovascular Surgery. 2005(04):247-248. Chinese.

\section{Publish your work in this journal}

The International Journal of General Medicine is an international, peer-reviewed open-access journal that focuses on general and internal medicine, pathogenesis, epidemiology, diagnosis, monitoring and treatment protocols. The journal is characterized by the rapid reporting of reviews, original research and clinical studies across all disease areas. The manuscript management system is completely online and includes a very quick and fair peer-review system, which is all easy to use. Visit http://www.dovepress.com/ testimonials.php to read real quotes from published authors. 